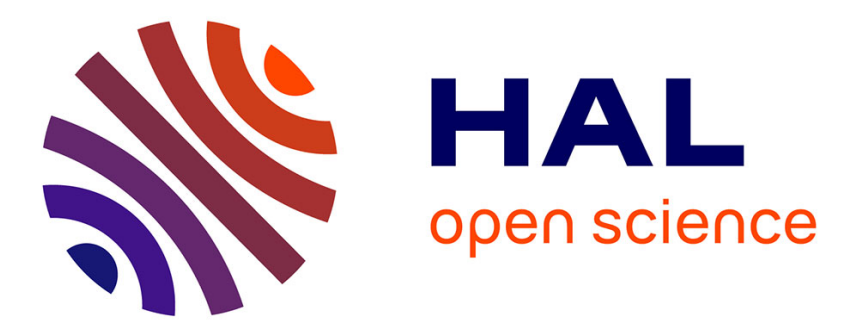

\title{
Adaptive densification of mobile networks: exploring correlations in vehicular and telecom traffic
}

\author{
Foroogh Mohammadnia, Marco Fiore, Marco Ajmone Marsan
}

\section{To cite this version:}

Foroogh Mohammadnia, Marco Fiore, Marco Ajmone Marsan. Adaptive densification of mobile networks: exploring correlations in vehicular and telecom traffic. 17th Annual Mediterranean Ad Hoc Networking Workshop (Med-Hoc-Net 2018), Jun 2018, Capri Island, Italy. pp.23-30. hal-01832488

\author{
HAL Id: hal-01832488 \\ https://hal.inria.fr/hal-01832488
}

Submitted on 7 Jul 2018

HAL is a multi-disciplinary open access archive for the deposit and dissemination of scientific research documents, whether they are published or not. The documents may come from teaching and research institutions in France or abroad, or from public or private research centers.
L'archive ouverte pluridisciplinaire HAL, est destinée au dépôt et à la diffusion de documents scientifiques de niveau recherche, publiés ou non, émanant des établissements d'enseignement et de recherche français ou étrangers, des laboratoires publics ou privés. 


\title{
Adaptive densification of mobile networks: exploring correlations in vehicular and telecom traffic
}

\author{
Foroogh Mohammadnia ${ }^{1}$, Marco Fiore ${ }^{2}$, Marco Ajmone Marsan ${ }^{1,2,3}$ \\ ${ }^{1}$ Electronics and Telecommunications Department, Politecnico di Torino, Italy \\ 2 CNR-IEIIT, Torino, Italy \\ ${ }^{3}$ IMDEA Networks Institute, Leganes, Spain
}

\begin{abstract}
In this paper we use real data to investigate the correlation in time and space between vehicular traffic and telecom traffic in cellular networks. The analysis of such a correlation is critical to assess the feasibility of cellular network architectures where densification is achieved with small-cell mobile base stations carried by vehicles. This is an attractive possibility for the provisioning of on-demand capacity through temporary dense small cell deployments where and when needed. Our results indicate that vehicular traffic at penetration rates expected for small-cell-carrying vehicles is much more bursty than telecom traffic. Yet, some correlations between the two emerge, even when considering an entire large metropolitan area. More importantly, correlations tend to be stronger in densely urbanized areas and during high-demand time periods, i.e., where and when radio access densification is most needed. Overall, our results indicate small-cell base stations carried by vehicles as a promising and cost-effective approach to dynamic provisioning of capacity in future-generation cellular networks.
\end{abstract}

Index Terms-Mobile network traffic; Vehicular traffic; Dense radio access network; Moving base station; Small cells.

\section{INTRODUCTION}

The use of large numbers of Small Cells (SCs) for the densification of Radio Access Networks (RANs) is regarded as a most promising approach for the provision of broadband services to large numbers of mobile network end-user terminals. However, the deployment of the SC base stations (BSs) necessary to make dense RANs a reality implies huge investments, which Mobile Network Operators (MNOs) are reluctant to approve in a period of declining profits.

A major reason for the high SC deployment cost is that peaks of bandwidth demand vary significantly in time and space [1], [2], thus making the SC utilization highly variable, and low on average. For instance, users tend to commute from home to work in the morning of working days, making business districts crowded during working hours. In this period, MNOs need high-capacity RANs, hence a dense presence of SCs, to be located in business areas. However, after work, users move out of their offices, so that the demand for RAN capacity becomes much lower in business districts, and many of the installed SCs become redundant. The opposite is true for residential areas, where capacity is mostly necessary in the evening, so that a dense RAN layout becomes useful then, not during working hours. In addition, during commuting and in the event of traffic jams or accidents, dense RAN layouts are needed where road traffic is congested. The cost of a RAN ISBN 978-3-903176-05-8 @ 2018 IFIP which is dense everywhere can be extremely high, and the associated long periods of low utilization imply a poor return on investment for the MNO.

A possible approach to make high RAN capacity available where and when needed is to use mobile SC BSs carried by vehicles. This approach leads to a network where not only end users, but also (part of) the network infrastructure is mobile. We name such an architecture a mobile mobile network, or MOMONET. The idea of mobile BSs is not new. Operators have exploited truck-mounted BSs for the quick provision of coverage in areas where service was not otherwise available, or where additional capacity was needed [3]. Some truckmounted BSs were periodically moved from beach areas in summer to skiing resorts in winter. However, in this paper we discuss a new, much broader use for mobile BSs, looking at the possible impact of vehicle-mounted SC BSs on the implementation of dense RANs based on SCs.

The feasibility of RAN densification with vehicle-mounted $\mathrm{SC}$ BSs raises a number of research questions that will require accurate investigation. For example: the selection of algorithms for the association of end users to BSs and for handover mitigation; the management of interference among transmissions from traditional BSs, from small cell BSs, and from end users; the selection of technologies for the implementation of small cell BSs on vehicles, and for the implementation of backhaul wireless connections; the reduction of the impact of the energy consumption of small cell BSs on vehicles power systems; the impact of self-driving cars.

Vehicles have the desirable property of moving together with end users ${ }^{1}$, so that in a business district during working hours we normally have both many end users and many vehicles. If a significant fraction of those vehicles carry a SC BS, a temporary dense SC deployment is created. Quite nicely, this temporary dense SC deployment will be recreated in residential districts when drivers return home with their cars, as well as in traffic jams.

Note that the correlation between the number of vehicles and the number of MNO end user terminals, hence between telecommunication traffic and vehicular traffic, was assumed in several works (see, e.g., [5] and [6]), starting with the Real Time Rome project of the MIT Senseable City Lab

\footnotetext{
${ }^{1}$ Here we mostly refer to humans, but vehicles can also be host to several connected devices; for instance, a recent study by HIS [4] predicts that by 2020 the fraction of connected cars in Europe will reach $50 \%$.
} 
[7], [8], but has never been carefully measured in real-world settings. In this paper, we aim at filling this gap. We use recent measurement data on two months of telecom traffic and vehicular traffic in the city of Milan, Italy, and evaluate their spatio-temporal correlation.

The rest of the paper is organized as follows. In Section II we briefly overview previous works on mobile BSs. In Section III we present our reference dataset. In Section IV we provide a preliminary exploration of the data. In Section V we illustrate and comment correlation results. In Section VI we investigate relationships further, by assessing the average distance between end user terminals and SC BSs carried by vehicles. Finally, Section VII concludes the paper.

\section{RELATED WORK}

The idea of using cars as active elements of a telecommunication network is not new. Several studies in the domain of vehicular networks considered the possibility of integrating a Wi-Fi Acces Point (AP) in cars, and possibly also a cellular interface, so that a vehicle can provide connectivity to the sourrounding cars. For example, [9] proposed the use of mobile vehicular gateways that exploit Wi-Fi for vehicleto-vehicle (V2V) communications and LTE for vehicle-toinfrastructure (V2I) communications. The study in [10] introduced the concept of Virtual APs, which allow extending the reach of roadside access points: vehicles that receive a message, store it, and rebroadcast it into non-covered areas. In [11]-[13], parked vehicles were exploited, in addition to roadside units, to improve the performance of video downloads and other services toward travelling cars. In [14], mobile nodes were used for the collection of data from sensors.

These approaches are different from a MOMONET, which leverages vehicles as a support for SC BSs of a dense RAN. MOMONETs allow the seamless integration of the vehiclemounted SC BSs with the "normal" macro- and micro-BSs at fixed locations; specifically, the moving SC BSs provide additional capacity, when and where needed, to the end users of an otherwise traditional RAN. End-user terminals can thus freely and transparently transfer their services between a normal and a vehicular BS. MOMONETs generalize the concept of early deployments of SC BSs onboard public transport vehicles to serve passengers in ultra-dense urban environments [15]-[18], and that of using vehicle-carried SC BSs in public safety networks [19]. A concept similar to MOMONET has also been briefly hinted at in recent works [20], [21], whose authors mention the possible use of $4 \mathrm{G}$ or $5 \mathrm{G}$ small cells within moving and parked cars, or on drones, so as to serve users both indoor and outdoor.

\section{THE DATASET}

We used a dataset that contains values of telecom and vehicular traffic during 61 days (44 working days and 17 weekend days) for the city of Milan, Italy, organized according to a subdivision of the area into 576 squares, as shown in Figure 1 [22]. The squares (actually, rectangles) have minimum size $220 \mathrm{~m} \times 330 \mathrm{~m}$, but sides can be 2, 4, 8 times longer, as

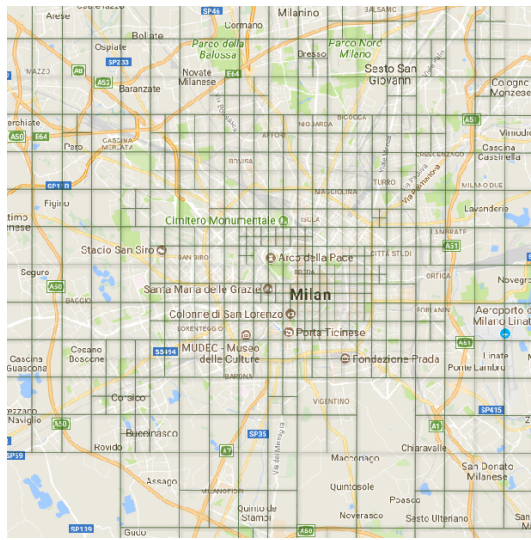

Fig. 1: The subdivision of the Milan area into 576 squares.

can be seen on the map. The time granularity of the dataset is 15 minutes (96 intervals per day). For each time interval, the dataset reports the total amount of mobile network data traffic (voice and data, no signaling) recorded in every square by Telecom Italia Mobile (TIM), the incumbent Italian MNO, as well as the GPS trajectories of active (i.e., not parked) vehicles tracked by InfoBlu (about 4 million, on a national scale), a large Italian infomobility services provider. The total number of time intervals is $61 \times 96=5,856$. Considering the number of area squares for which data are reported separately, we have a total of $3,373,056$ data points.

It is important to remark that the penetration rates of the two measurement technologies are different. While the telecom traffic in the dataset corresponds to more than one third of the overall mobile network demand, the fraction of the monitored vehicles is less than $1 \%$, as it mainly encompasses probe vehicles and commercial fleets monitored by InfoBlu. We stress that these settings are consistent with the goal of our investigation: we expect a small percentage of vehicles to act as SC BS carriers, and would like them to cover the largest fraction possible of the total telecom traffic.

\section{DATA EXPLORATION IN REPRESENTATIVE CASES}

We start by showing the average telecom and vehicular daily traffic patterns in a representative selection of squares. We consider squares of different types, based on several major land uses in the area: a) residential, b) business, c) train station, d) airport, and e) soccer stadium. Figure 2 shows the average telecom and vehicular traffic patterns for all days of the week for the five selected squares (telecom traffic in the left plots and vehicular traffic in the right plots). We observe a number of interesting features in both telecom and vehicular daily traffic patterns. First of all, while in telecom traffic clear behaviors exist, the vehicular traffic is highly irregular. In a residential area, plots (a) and (b) in Figure 2, we can clearly see that the telecom traffic is very low at night (roughly from 2 am to $7 \mathrm{am}$ ), it increases (sharply early in the morning, then more mildly) until $10 \mathrm{pm}$, and finally starts declining again. No big differences exist among days, except for the fact that the traffic on Sundays is higher during the night and around midday. 


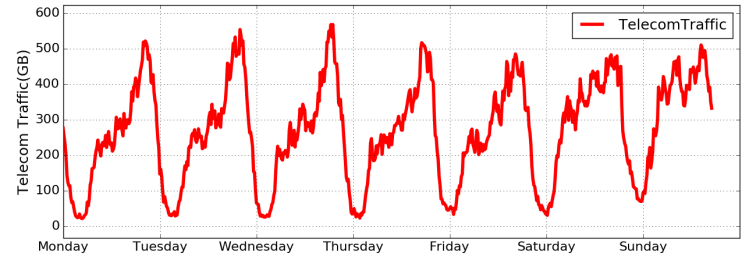

(a)

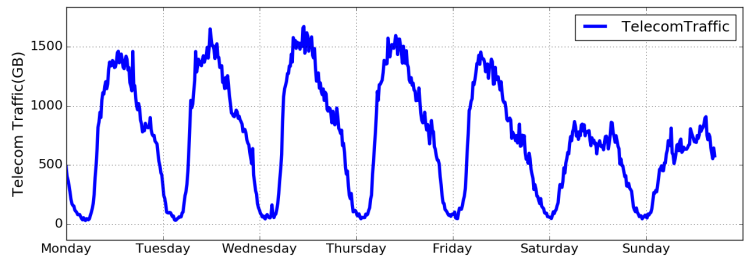

(c)

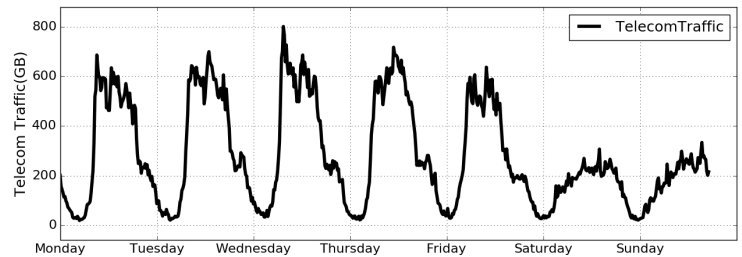

(e)

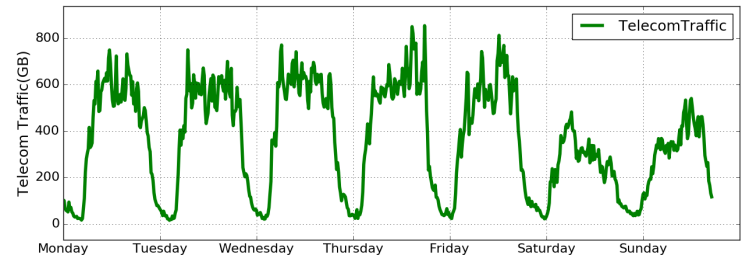

(g)

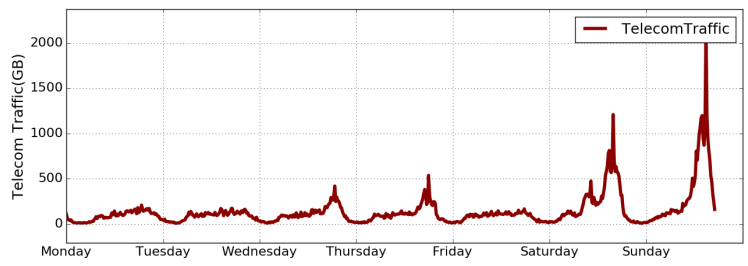

(i)

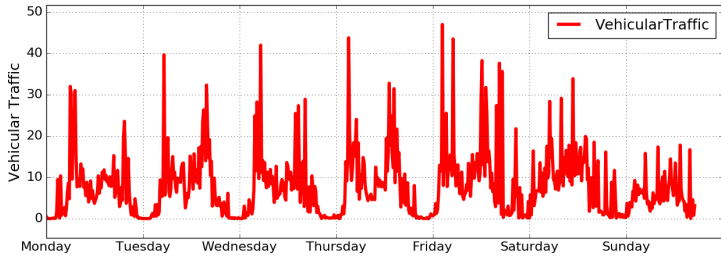

(b)

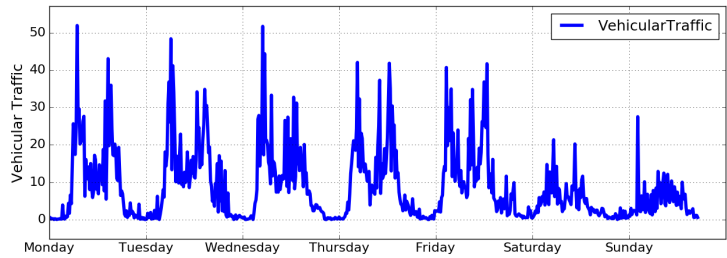

(d)

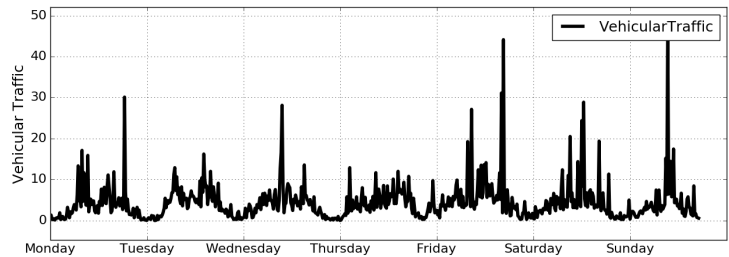

(f)

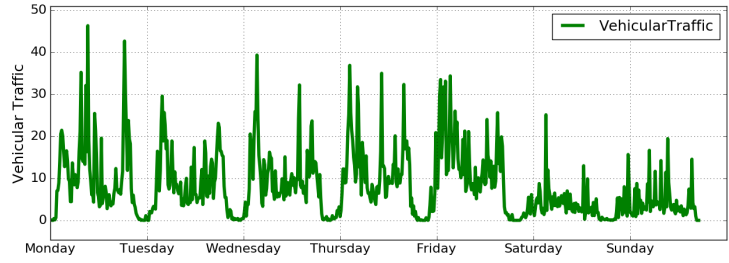

(h)

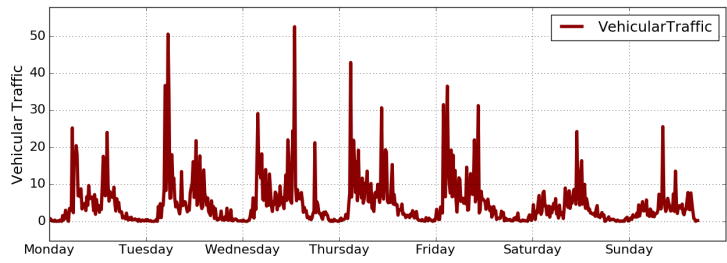

(j)

Fig. 2: Average telecom and vehicular traffic (left and right, respectively) for all days of the week in selected square areas. Plots (a) and (b) refer to a residential area, plots (c) and (d) to a business area, plots (e) and (f) to a train station, plots (g) and (h) to an airport, and plots (i) and (j) to a soccer stadium.

Vehicular traffic exhibits instead many ups and downs, with the highest peaks between 7 am and $10 \mathrm{am}$; also, vehicular traffic on Sundays is lower than in other days.

In a business area, plots (c) and (d) in Figure 2, telecom traffic is very low at night (roughly from 2 am to $7 \mathrm{am}$ ), it increases sharply around 8 am in working days, and then gradually decreases after $2 \mathrm{pm}$. The peak of telecom traffic in weekends is about half the one in working days. Vehicular traffic again exhibits high variability, with peaks at around 8 am and between $3 \mathrm{pm}$ and $5 \mathrm{pm}$, and is lower on Sundays.

In a train station area, plots (e) and (f), telecom traffic is once more very low at night, then sharply increases around 8 
am in working days, and remains close to its peak value from 9 am to $9 \mathrm{pm}$, then sharply decreases. The peak of telecom traffic in weekends is about one third of that in working days. Vehicular traffic is very bursty, as usual, peaking in the afternoon and evening.

In an airport area, plots $(\mathrm{g})$ and $(\mathrm{h})$, telecom traffic is very low at night, sharply increases around $5 \mathrm{am}$, and remains close to its peak value from 8 am to $10 \mathrm{pm}$, then sharply decreases. The peak of telecom traffic in weekends is about two-thirds of the one in working days. Vehicular traffic as usual exhibits many ups and down along most of the day.

Finally, in a soccer stadium area, plots (i) and (j), telecom traffic exhibits very high peaks around $9 \mathrm{pm}$ in Saturdays and Sundays, when soccer matches are played (and concerts take place). Otherwise, traffic is close to zero. Vehicular traffic is once more irregular, peaking at around 7 am and early in the afternoon.

The inspection of the raw data about telecom and vehicular traffic that we presented so far does not provide many clues to justify correlation; rather, it reveals that telecom traffic dynamics vary widely in space and time, and our reference vehicular traffic is generally much more bursty than telecom traffic. This latter effect is mainly due to the reduced fraction of vehicles carrying SC BSs that will characterize MOMONET deployments.

We provide a more global overview of the evolution of vehicular and telecom traffic in Figure 3. There, the variation of traffic in space is visualized through heat maps, where for each square the color indicates the (telecom or vehicular) traffic level (dark blue is lowest and dark red is highest - the color scale is logarithmic). The different plots portray heat maps for a same day (March 2, 2015) at five different times: $5 \mathrm{am}, 10 \mathrm{am}, 3 \mathrm{pm}, 8 \mathrm{pm}$ and midnight. We can see from these plots (and for similar ones at all time intervals in the day) that telecom traffic, in comparison to vehicular traffic, rises earlier in the morning, then it remains high later in the evening, and is more pervasive over the entire Milan area. Also the simple visual inspection of the geographical distribution of vehicular and telecom traffic above does not seem to highlight evident correlations, except for the fact that traffic peaks coincide with the city center in both cases.

\section{CORRELATION ANALYSiS}

As the qualitative analysis in Section IV did not reveal evident relationships, we carry out a comprehensive quantitative study of the correlations between telecom and vehicular traffic. Specifically, we separately explore the spatial, temporal, and spatiotemporal dimensions of the problem.

\section{A. Spatial correlation}

The results about correlations from a spatial perspective are summarized by the scatterplots in Figure 4. There, the abscissa refers to the telecom traffic value, and the ordinate reports the vehicular traffic value for each dot corresponding to one square in the Milano area (thus 576 dots), and traffic values are averaged over all time intervals of working days
(Monday through Friday; $44 \times 96$ instances - top plot) and weekends (Saturday and Sunday; $17 \times 96$ instances - bottom plot). Therefore, Figure 4 outlines correlations emerging across the geographical areas in the target metropolitan region. Plots also show the least square linear regression line, whose slope is 0.018 for working days and 0.016 for weekends.

The positive slope values indicate that a positive correlation exists between telecom and vehicular traffic across space, despite the dispersion of points around the regression line.

\section{B. Temporal correlation}

Correlations in the time dimension are considered in Figure 5 , where each dot of the scatterplots represents one daily time interval (for a total of 96 dots). Results are aggregated over the whole metropolitan area: the coordinates of each dot thus represent the average telecom and vehicular traffic in the time slot, averaged over 576 squares and either 44 working days in plot (a) or 17 weekend days in plot (b). The shape of these plots is quite interesting, if we look at them while considering the sequence of time slots. For this, plots (c) and (d) report the same data as plots (a) and (b), respectively, in a 3D plot, where the $\mathrm{z}$-axis represents time of the day. We see that a significant amount of telecom traffic is present at 12:15 am, while vehicular traffic is close to zero. Telecom traffic then declines, and both types of traffic are at their minimum around $3 \mathrm{am}$. Vehicular traffic then starts to increase, while telecom traffic remains low (time runs clockwise along the dot sequences in Figure 5, plots (a) and (b)). Then, telecom traffic increases, and remains high until late in the evening, when vehicular traffic has become low. Finally, also telecom traffic dies out. Note that the effects are more pronounced during working days than in weekends.

Linear regression lines highlight a positive correlation also in this case, although points are typically far from the behavior predicted by a simple linear model. The limited goodness of fit can be imputed to the temporal offset between the high activity periods of vehicular and telecom traffic explained above.

\section{Spatio-temporal correlation}

We delve deeper in the analysis by disaggregating traffic over both space and time. Figure 6 shows plots where each dot refers to one square in one 15-minute interval of the day (hence $576 \times 96=55,296$ dots in each plot). The coordinates of each dot are the average telecom and vehicular traffic recorded during either 44 working days or 17 weekend days. Also in this case, the least square linear regression lines have positive slopes, equal to 0.014 in the case of working days, plot (a), and 0.013 in the case of weekends, plot (b), which entail positive correlation for both. However, dots are now even more scattered over the quadrant, indicating that the linear regression is not representative of many combinations of squares and daytimes.

\section{When and where is correlation higher?}

The results above yield the following conclusions: i) there exist a general positive correlation between vehicular and 


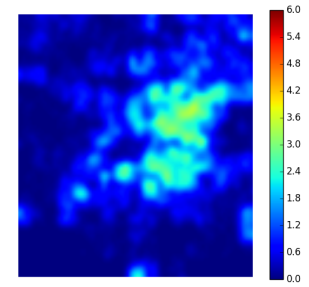

(a)

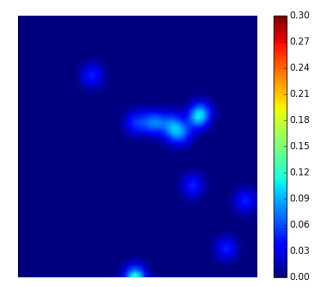

(f)

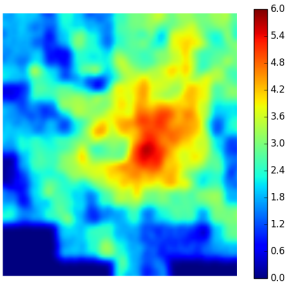

(b)

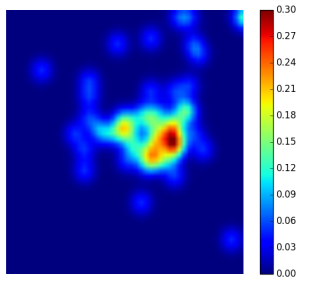

(g)

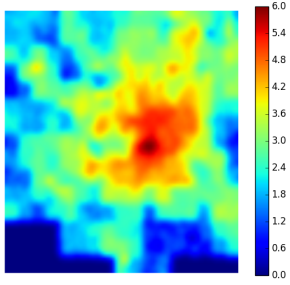

(c)

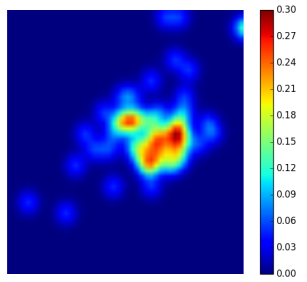

(h)

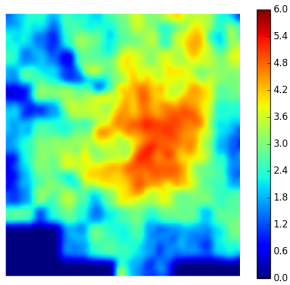

(d)

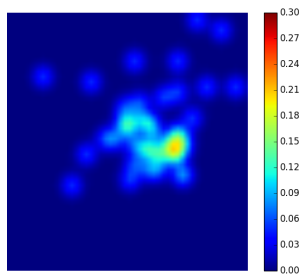

(i)

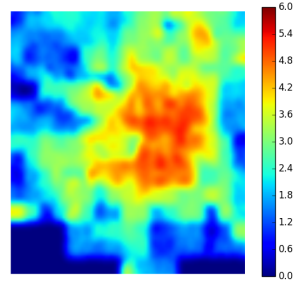

(e)

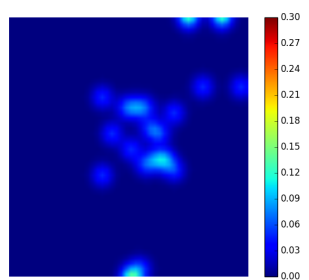

(j)

Fig. 3: Heatmaps of Telecom (first row) and vehicular (second row) traffic on March 2, 2015. The first column shows data at $5 \mathrm{am}$; the second column at $10 \mathrm{am}$, the third at $3 \mathrm{pm}$, the fourth at $8 \mathrm{pm}$, the last at midnight. The plotted area is about $18 \times 21 \mathrm{~km}$.

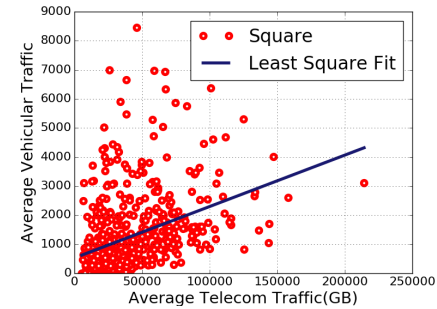

(a)

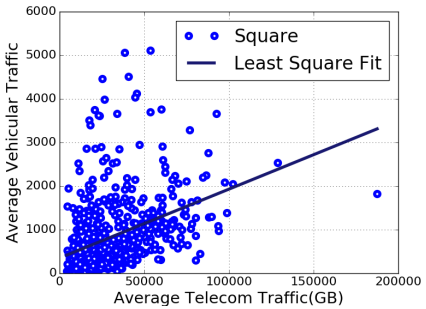

(b)
Fig. 4: Correlation between average telecom and average vehicular traffic. Plot (a) refers to 44 working days and plot (b) to 17 weekend days.

telecom traffic, but ii) the correlation is low, due to a high variability of relations over time and space. A sensible question is then whether some hours and geographical areas show especially strong correlations, and are thus suitable periods and locations for MNOs to take advantage of the MOMONET paradigm. To answer this question, we leverage three different correlation coefficients:

- The Pearson product-moment correlation coefficient $r$, defined as:

$$
r=\frac{n \sum_{i=1}^{n} x_{i} y_{1}-\left(\sum_{i=1}^{n} x_{i} \sum_{j=1}^{n} y_{j}\right)}{\sqrt{\left[n \sum_{i=1}^{n} x_{i}^{2}-\left(\sum_{i=1}^{n} x_{i}\right)^{2}\right]\left[n \sum_{i=1}^{n} y_{i}^{2}-\left(\sum_{i=1}^{n} y_{i}\right)^{2}\right]}}
$$

where $n$ is the number of observations for both telecom and vehicular traffic, and $x_{i}, y_{i}$ are the telecom and vehicular traffic observations, respectively.

- The Spearman rank correlation coefficient $s$, defined as:

$$
s=1-\frac{6 \sum_{i=1}^{n} y_{i}^{2} d_{i}^{2}}{n\left(n^{2}-1\right)}
$$

where $d_{i}$ is the difference between the ranks (ordered positions) of corresponding telecom and vehicular traffic samples.

- The Kendall rank correlation coefficient q, defined as:

$$
q=\frac{n_{c}-n_{d}}{n(n-1) / 2}
$$

where $n_{c}$ is the number of corresponding telecom and vehicular traffic samples with the same rank and $n_{d}$ is the number of corresponding telecom and vehicular traffic samples with different rank. We first investigate which are the periods during the day when the correlation is higher. Figure 7 (a) shows the curves of the Pearson correlation coefficient for the average working day and for the average weekend day. The curves for the Spearman and Kendall correlation coefficients are not reported, but are very similar. We observe that correlation is low overnight in working days and in the early morning of 


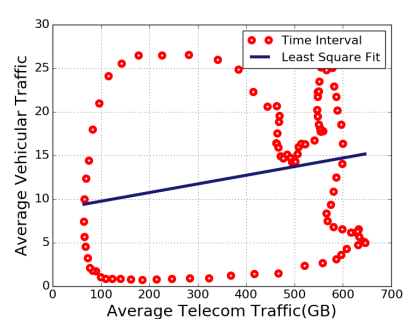

(a)

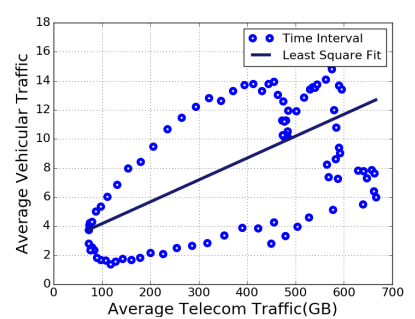

(b)

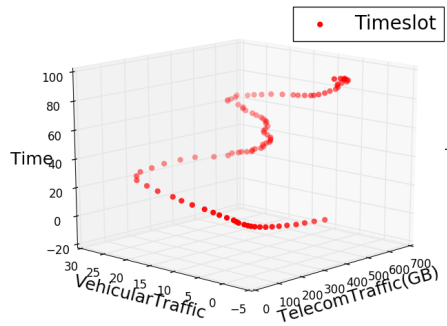

(c)

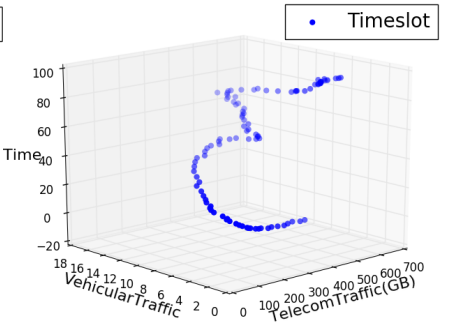

(d)

Fig. 5: Correlation between average telecom and average vehicular traffic in each timeslot over 44 working days in plot (a) and over 17 weekend days in plot (b); plots (c) and (d) report the same data as (a) and (b), with time increasing along the z-axis.

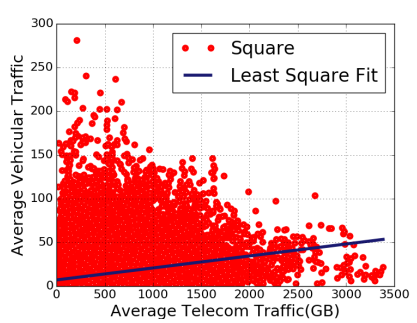

(a)

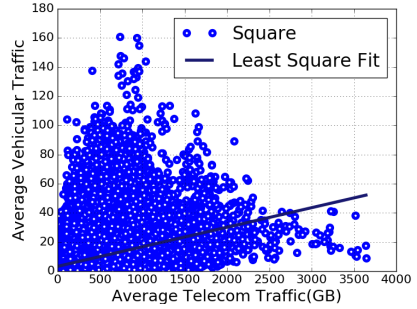

(b)

Fig. 6: Correlation between average telecom and average vehicular traffic in each square in each timeslot over 44 working days in plot (a) and over 17 weekend days in plot (b).

weekends. On the contrary, the correlation coefficient values are higher - in the range from 0.3 to 0.5 - during high-demand periods, i.e., in working and evening hours. These are good news, since the correlation is low in the night periods, when the demand for mobile services is typically low, and network densification is not necessary. Instead, MOMONETs are the most effective exactly when densification is needed, that is, in presence of mobile network traffic demand peaks.

Concerning the geographical dispersion of correlation, Figure 7 (b) portrays the cumulative distribution functions (CDFs) of the values of the three correlation coefficients for all spatial squares in the considered metropolitan area. We can see that negative correlations are limited to a few cases, and correlation coefficient values are positive in the large majority (90-95\%) of areas. Differences across coefficient types are also small, as the Pearson and Spearman correlation coefficients yield very similar CDFs, while the $\mathrm{CDF}$ of the Kendall correlation coefficient values indicates some prevalence of lower values of correlation coefficients. However, the important observation is that the three CDFs confirm the existence of spatial heterogeneity of correlation: depending on the considered square, the correlations vary significantly, and can reach values as high as 0.84 .

In order to understand where such high-correlation squares

lie, Figure 7 (c) shows a heatmap where the color of each square corresponds to the Pearson correlation coefficient computed over an average day. We can clearly see that correlation is stronger in the city center. On the one hand, this is expected, since both vehicular and telecom traffic are higher in those squares, hence they follow more predictable patterns. On the other hand, and more importantly, those are also the squares where the demand for radio access network capacity is higher, hence densification is needed the most. We conclude that, in our reference scenario, MOMONETs prove to be mainly exploitable not only when, but also where network densification is needed the most.

\section{AVerage Distance}

As a final complement to our analysis, we comment on the average distance between SC BS-equipped vehicles and the location of end user terminals that can use the SC BSs carried by vehicles to transmit and receive mobile network traffic. Since our dataset reports exact vehicle positions within each square, but only the origin square (and not the exact end user terminal location) for telecom connections, we randomly place end user terminals within the area covered by each square. Of course, this is an approximation, but we have no information to drive a more realistic placement of end users. The average distance between the end user terminal and the closest SC BS (the one to which the end user terminal normally associates) is a metric of great interest for MOMONETs, since it is a proxy for the connection data rate.

Results in Figure 8 (a) show the evolution of such average distance over time, averaged over all spatial squares and averaged over all 61 days. The average distance is high (it can grow over $190 \mathrm{~m}$ ) in periods of low telecom and vehicular traffic, and it stays at around $120 \mathrm{~m}$ during periods characterized by high network traffic, between 7 am and 6 pm roughly. These distances risk to make connections to SCs frequently unviable, due to poor signal strengths (consider that the power emitted by a SC is typically less than $1 \mathrm{~W}$, and reach depends on propagation conditions, but normally is less than $100 \mathrm{~m})$.

The same observation holds when considering the geographical distribution of distances, averaged over the whole day. In 


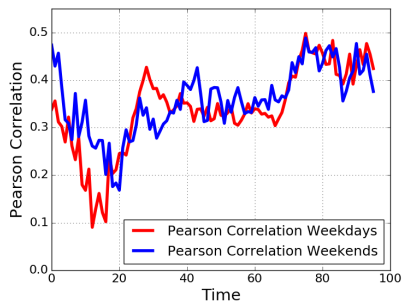

(a)

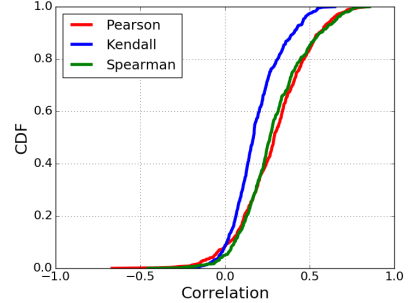

(b)

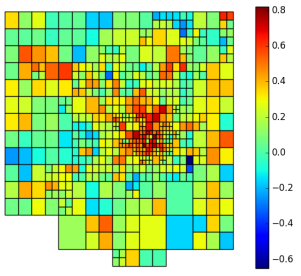

(c)

Fig. 7: Pearson correlation coefficient between average telecom and vehicular traffic over all squares and over 44 working days and 17 weekend days in plot (a); cumulative distribution function of the correlation coefficients between average (over 61 days and over timeslots) telecom and vehicular traffic in each square in plot (b); Pearson correlation coefficient heat map between telecom and vehicular traffic for each Milano square, averaged over 61 days in plot (c).

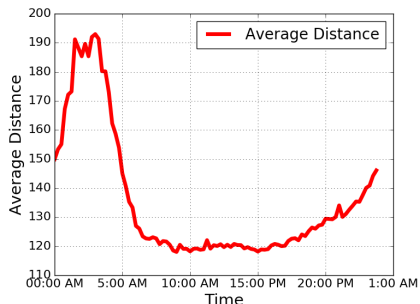

(a)

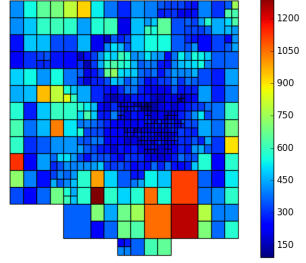

(b)

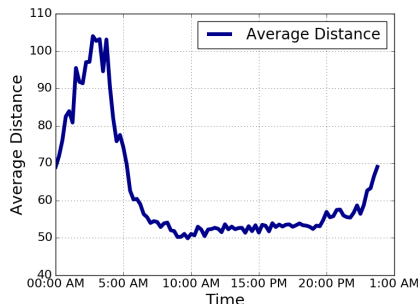

(c)

Fig. 8: Average distance between vehicles and origins of telecom traffic versus time of day averaged over 61 days and over all Milano squares in plot (a); average distance between vehicles and origins of telecom traffic for all square areas over one day (March 2, 2015) in plot (b); and average distance between vehicles and origins of telecom traffic versus time of day for 158 squares in the center of Milan averaged over 61 days in plot (c).

plot (b) in Figure 8, we show for all 576 squares that cover Milan the average distance between the position of vehicles and the connection origin. The shortest average daily distances are found in the city center and are around $120 \mathrm{~m}$, while the longest average daily distances are observed in peripheral squares and grow up to around $200 \mathrm{~m}$. Again, SCs are hardly usable under such distances.

However, when focusing on the city center, the outcome is different. Plot (c) in Figure 8 depicts the average distance between the position of end user terminals and the closest SC BS carried by a vehicle, in the 158 squares in the center of Milan averaged over all 61 days. Between 9 am and $8 \mathrm{pm}$ average distances are around $50 \mathrm{~m}$, so that in this portion of the city we can expect a large fraction of terminals to be within reach of a SC BS carried by a vehicle. These values nicely complement the results in Section V, and confirm that MOMONETs represent a suitable network paradigm to enable the adaptive densification of radio access envisioned for nextgeneration $5 \mathrm{G}$ systems, at least in the highly urbanized areas where mobile network capacity will be needed the most.

\section{CONCLUSIONS}

We used real traces of vehicular traffic and telecom traffic to investigate the correlation in time and space between the two types of traffic. The motivation for the study lies in the assessment of the feasibility of cellular network architectures where densification is achieved with small-cell mobile base stations carried by vehicles, according to a novel mobile mobile network, or MOMONET, architecture. Our results prove that correlation between vehicular and telecom traffic is low when we consider an entire metropolitan area, but becomes stronger when we look at the areas and the time periods where and when densification is necessary to serve peaks of telecom traffic. Similar considerations hold when looking at the distance between mobile SC BSs and the users they could serve. Ultimately, these observations support small-cell base stations carried by vehicles, and the associated MOMONET paradigm, as a promising and cost-effective approach to the adaptive densification of cellular networks.

\section{ACKNOWLEDGMENTS}

This work was supported by the Siebel Energy Institute through the Seed Grant Award 59/2016. This research received funding from the People Programme (Marie Curie Actions) of the European Union's Seventh Framework Programme under REA grant agreement n.630211, ReFleX. 


\section{REFERENCES}

[1] A. Furno, D. Naboulsi, R. Stanica and M. Fiore, "Mobile Demand Profiling for Cellular Cognitive Networking," IEEE Transactions on Mobile Computing, n. 99, pp. 1-1, 2016.

[2] A. Furno, R. Stanica and M. Fiore, "A comparative evaluation of urban fabric detection techniques based on mobile traffic data," in IEEE/ACM International Conference on Advances in Social Networks Analysis and Mining, Paris, 2015.

[3] DAEL, "http://www.dael.com/en/telecom/cell-on-wheels," [Online].

[4] IHS, "IHS Connected Car consumer survey finds strong appetite for ADAS features and autonomous cars," [Online]. Available: https://technology.ihs.com/580128/ihs-connected-car-consumer-surveyfinds-strong-appetite-for-adas-features-and-autonomous-cars.

[5] A. Janecek, D. Valerio, K. A. Hummel, F. Ricciato, and H. Hlavacs, "The Cellular Network as a Sensor: From Mobile Phone Data to Real-time Road Traffic Monitoring," IEEE Transactions on Intelligent Transportation Systems, vol. 16, no. 5, 2015.

[6] N. Caceres, L. M. Romero, F. G. Benitez and J. M. del Castillo, "Traffic flow estimation models using cellular phone data," IEEE Transactions on Intelligent Transportation Systems, vol. 13, no. 3, 2012.

[7] MIT, "http://senseable.mit.edu/," [Online].

[8] MIT, "http://senseable.mit.edu/realtimerome/," [Online].

[9] N. M. Sadek, H. H. Halawa, R. M. Daoud and H. H. Amer, "A Robust Multi-RAT VANET/LTE for Mixed Control \& Entertainment Traffic," Journal of Transportation Technologies, n. 5, pp. 113-121, 2015.

[10] N. Frangiadakis, D. Cmara, F. Filali, A. A. F. Loureiro and N. Roussopoulos, "Virtual Access Points for Vehicular Networks," in MOBILWARE 2008, 1st International Conference on MOBILe Wireless MiddleWARE, Operating Systems, and Applications, Innsbruck, Austria, 2008.

[11] F. Dressler, P. Handlex and C. Sommer, "Towards a Vehicular Cloud Using Parked Vehicles as a Temporary Network and Storage Infrastructure," in WiMobCity14, Philadelphia, PA, USA, 2014.

[12] F. Malandrino, C. E. Casetti, C.-F. Chiasserini, C. Sommer and F. Dressler, "Content Downloading in Vehicular Networks: Bringing Parked Cars Into the Picture," in 23rd PIMRC 2012, IEEE International Symposium on Personal, Indoor and Mobile Radio Communications, Sydney, Australia, 2012.

[13] F. Malandrino, C. Casetti, C. F. Chiasserini, C. Sommer and F. Dressler, "The Role of Parked Cars in Content Downloading for Vehicular Networks," IEEE Transactions on Vehicular Technology, vol. 63, n. 9, pp. 4606-4617, 2014.

[14] D. Borsetti, C. Casetti, C.-F. Chiasserini, M. Fiore and J. M. BarcelOrdinas, "Virtual data mules for data collection in road-side sensor networks," in Second International Workshop on Mobile Opportunistic Networking (MobiOpp '10), New York, NY, USA, 2010.

[15] Y. Sui, I. Guvenc and T. Svensson, "On the deployment of moving networks in ultra-dense urban scenarios," in 1st International Conference on 5G for Ubiquitous Connectivity, Akaslompolo, 2014.

[16] A. Panno and D. Mastrosimone, "New challenge: Moving network based on mmWave technology for 5G era," in 2015 International Conference on Computer, Information and Telecommunication Systems (CITS), Gijon, 2015.

[17] S. Jangsher and V. O. K. Li, "Resource Allocation in Moving Small Cell Network," IEEE Transactions on Wireless Communications, vol. 15, $\mathrm{n}$. 7, pp. 4559-4570, 2016.

[18] H. Yasuda, A. Kishida, J. Shen, Y. Morihiro, Y. Morioka, S. Suyama, A. Yamada, Y. Okumura and T. Asai, "A Study on Moving Cell in 5G Cellular System," in 2015 IEEE 82nd Vehicular Technology Conference (VTC2015-Fall), Boston, 2015.

[19] M. Shin, S. T. Shah, M. Y. Chung, S. F. Hasan, B.-C. Seet and P. H. J. Chong, "Moving small cells in public safety networks," in 2017 International Conference on Information Networking (ICOIN), Da Nang, 2017.

[20] S. Andreev, V. Petrov, M. Dohler and H. Yanikomeroglu, "Future of Ultra-Dense Networks Beyond 5G: Harnessing Heterogeneous Moving Cells," https://arxiv.org/abs/1706.05197, 2017.

[21] M. Pous-Fenollar and P.Fertl, "Mobile Crowdcell," in MWC, Barcelona, 2016.

[22] http://www.telecomitalia.com/tit/en/bigdatachallenge.html 